\title{
A model to represent human activities in farming systems based on reactive situated agents
}

\author{
$\underline{\text { Z. Afoutni }}^{\text {a,b }}{ }^{\text {, R. Courdier }}{ }^{\text {b }}$ and F. Guerrin ${ }^{\text {a,c }}$ \\ ${ }^{a}$ CIRAD, UR Recyclage et risque, F-97408 Saint-Denis, Reunion, France \\ ${ }^{b}$ Université de la Réunion, Laboratoire d'Informatique et Mathématique, F-97490 Ste-Clotilde, Reunion, \\ France \\ ${ }^{c}$ INRA, UR Biométrie et Intelligence artificielle, F-31326 Castanet-Tolosan, France \\ Email: zoubida.afoutni@,cirad.fr
}

\begin{abstract}
This paper proposes a model to represent and simulate human activities in farming systems. Our definition of the activity concept (otherwise said 'action') stems from the 'situated action' theory (Suchman, 1987). Action may be summarized simply as 'what people are actually doing'. Therefore, we characterize action according to four dimensions: actors (persons or machines engaged in activity), time (start date, end date and duration), space (locations where action occurs) and the interaction with the actor's environment. To take into account all these dimensions, our model is based on: (i) an 'agent' approach, (ii) a mathematical formalism to represent action over time (Guerrin, 2009) and (iii) a discrete representation of space inspired from cellular automata. Our aim is to apply this model to representing the human component of agricultural systems, i.e. the farming practices.
\end{abstract}

Space is modelled as a regular grid, each cell being controlled by a specific autonomous abstract 'agent', in the sense of multiagents systems (MAS), called 'cell-agent'. Since activity depends, according to the situated action paradigm, on its context of occurrence and continuously involves interactions with the environment, we focused on the concepts of stigmergy and affordance to carry the information necessary to realize actions and on the reactive agent approach. The radical notion of reactive agent postulates that agents' actions are reactions to stimuli. But if several stimuli motivate an agent to execute various actions at the same time, how should this agent act? In addition, various constraints to be dealt with are imposed by our application domain (farming systems), namely temporal constraints cell-agents must consider for acting. Consequently, our model, although based on a stimulus-response mechanism, involves also a process, kept as simple as possible, allowing an agent to select the most appropriate action when several actions are concurrently possible.

Cell-agent actions are realized through 'objects' representing, unconventionally, the real actors (e.g. the farmer possibly using equipments, animals, etc.). An 'object' is a non-autonomous entity that receives from the cell-agent where it is located the command of actions it must execute. A cell-agent can perceive, at any time, what happens in its neighbouring cells and which are the objects these cells host. In case a cell-agent needs a resource not located in a nearby cell, it can possibly get it by propagating its request through its neighbours.

Action is represented by a binary function of time (Guerrin, 2009) and implemented by specific objects called 'actuators' that are actually directed by the cell-agents they rely on. Action starts and ends according to events detected on processes observed by the cell-agents in their environment, provided they can access the necessary resources, among which actuators endowed with relevant abilities. The course of action is subject to a set of conditions that must remain satisfied for the action go to its end. In the meanwhile, it may be interrupted, resumed or cancelled.

The originality of this work lies in that actual 'actors' (i.e. persons, animals, machines, etc.) are represented as non-autonomous 'objects' and not as autonomous 'agents', as it is generally the case in other works (e.g., Sierhuis et al., 2006). These objects cannot act by themselves. They just follow the instructions given by the cell-agents they rely upon. Our model allows so one to represent the behaviour of real 'situated' actors as if it were the environment that dictated them what to do. Doing this way complies with the situated action theory.

Keywords: Situated action, Stigmergy, Affordance, Agent-based modelling, Agricultural systems. 


\section{INTRODUCTION}

Modelling human activities in farming systems has been approached by few authors as Martin-Clouaire and Rellier (2009). Action is represented in such models based on a 'planning' approach: an action plan, used as model input, specifies the set of possible actions and their organization over time. This plan is possibly adjusted while being executed to account for unpredictable events in the actors' environment. Our aim is also to represent human activities in farming systems. For this, we use another action theory called 'situated action' (Suchman, 1987). This approach emphasizes the role of 'situations' in which actors are embedded in the accomplishment of action. As outlined by Guerrin (2009): "performing an action is created by and creates itself the situation it is a part of; any action is singular (no two actions are the same) and contingent (it may happen or not according to the situation encountered)". We investigate alternative ways to represent action in agricultural systems to remedy to the inconveniences of the planned one. More precisely we want to model (i) what is done? (ii) when? (iii) where? (iv) by whom? We thus try to represent real activities, taking into account their features of being situated both in time and space and realized by some actors. A first action modelling framework based on situated action was developed by Guerrin (2009). This model answers the first two questions (what? and when?). Our current aim is to extend this model by integrating the spatial dimension of realization of action (where?) and an explicit representation of actors (whom?). Hence, our modelling effort tries to integrate a mathematical formalism representing action over time, a discrete representation of space inspired from cellular automata and a 'multiagents system' (MAS) approach.

This paper is organized as follows. In Section 1 we define our representation concepts and the formal representation of action. Section 2 is devoted to present our multiagents system. In Section 3, we present an example of application based on our concepts and multiagents representation.

\section{ACTION REPRESENTATION}

In the core of our modelling ontology is the notion of "action", performed by a human or a machine "actuator" in a particular "situation" which corresponds to the actuator's environment state at a given time and in a specified portion of space. An action usually involves interactions between the actuator and its environment. Given a set of actions, managing their possible interdependencies is called coordination. Without coordination a group of actors can quickly degenerate into a collection of separate individuals with chaotic behaviour. Coordination can be explicit or implicit. Our model is based on implicit coordination and, more precisely, on the two concepts of "stigmergy" and "affordance" to implement this coordination mode.

\subsection{Action}

Situated action defines action as emerging from the interactions among actors and with their environment. Rather than abstracting action by a plan tracing its course independently from its reality, we found better to study how actors use the circumstances they encounter to exhibit intelligent behaviours (Suchman, 1987). It is true that, sometimes, we construct mental models of "what we should do", based on our current knowledge, intented activities and anticipation of future events. This led to the Belief-Desire-Intention (BDI) model (Bratman, 1987) that became famous in the MAS community. Although relatively widespread, this BDI model remains theoretical as it does not fit with real observations made, for example, by ethnomethodologists or ergonomists in real settings (Agre, 1995; Johnston, 1998). Johnston (1998) argued that "It is one thing to think about doing something and quite another to do it". According to him, the planning approach fails to account for the situatedness and embodiment of real agents in physical settings. Although it may be a good theory of thinking ex-post about actions, it does not fit with representing on-going action.

Our conception of action, in contrast, is inspired from the situated action theory (Suchman, 1987) as we aim at representing human activities in complex farming systems at the operation level rather than at the tactical or strategic ones. More precisely, our focus is on what is actually performed by actors such as humans, animals and machines. With this respect, an action is a dynamical process that evolves according to the situations it encounters. The result of an action modifies its situation which in turn influences the next or surrounding actions. It is situated both in time (an action has a start date, an end date and a duration) and in space (it occurs somewhere). Guerrin (2009) proposed a mathematical formalism allowing one to represent action situated in time.

Every action $A$ is represented by a binary function of time (Eq. 1):

$$
S_{A}(t)= \begin{cases}1 & \text { if } C_{A}(t) \\ 0 & \text { otherwise }\end{cases}
$$


where $C_{A}(t)$ is a conjunction of conditions represented by logical propositions evaluated to true or false whether its value is respectively 1 or 0 . It actually represents the situation in which the action occurs.

The start $\left(\mathrm{t}^{-}\right)$and end $\left(\mathrm{t}^{+}\right)$dates and the duration $\left(\tau_{A}(t)\right)$ of an action are also functions of time (Eq. 2) determined according to a condition $P_{A}^{-}(t)\left(\operatorname{resp} . P_{A}^{+}(t)\right)$ :

$$
t_{A}^{ \pm}(t)=\left\{\begin{array}{l}
t \quad \text { if } \quad P_{A}^{ \pm}(t) \\
t_{A}^{ \pm}\left(\max \left(0, t-\tau_{S}\right)\right) \quad \text { otherwise }
\end{array}\right.
$$

where $\tau_{S}$ is the simulation time-step, $P_{A}^{-}(t)$ (resp. $\left.P_{A}^{+}(t)\right)$ is a logical proposition function of time, specified according to any process (or clock, schedule, affordance, etc.) on which events relevant to trigger or stop an action are possibly detected.

\subsection{Situation}

As 'situation' is the focus of the situated action theory, one can ask what does exactly this term mean. According to Visetti (2002), any action is always socially and physically situated, which is essential to interpret it. From this, we can characterize a situation by a physical context which denotes the actors' environment. In our case, this context is made of a physical space (viewing farming systems as sets of cultivated land plots and places holding buildings, material stocks, etc.) and all the necessary objects and resources involved in achieving activities, namely various kind of actuators (humans, animals, machinery, etc.) and the stocks of materials involved as inputs or outputs in the production processes (feeds, fertilizers,...). All these objects are characterised by properties describing their state. For example, the properties of a land plot can be the climatic conditions (temperature, pluviometry, etc.), the crop which it supports, its soil characteristics (quantity of nitrogen, hydromorphy, etc.) and so on. Although the social context of action should also be accounted for, we did not consider it at the moment. Hence an agent's situation is made of its current perceptions of its physical context while acting at a specific time and place.

\subsection{Coordination}

Coordination allows the dependencies between actions to be managed in order to assure a coherent system's behaviour. Malone and Crowston (1994) have characterized four kinds: resource sharing, producer-consumer relationship, simultaneity constraints, task-subtask dependencies. As mentioned above, two ways of implementing coordination are found in the literature: explicitly, by the intermediary of protocols (plans, procedures, rules, etc.), or implicitly, by perceiving and reacting to changes in the environment. Implicit coordination, the most coherent way with respect to the situated action paradigm, is generally based on two concepts: stigmergy and affordance.

\section{Stigmergy}

This concept was coined by the biologist Pierre-Paul Grassé (1959) investigating the behaviour of social insects. A form of coordination was identified based on marks of odoriferous substances called pheromones deposited by ants in their environment. Pheromones modify the physical context for other ants and determine their future actions. Stigmergy, as an indirect communication means, can be mapped to the agent approach according to the following principles:

- Each agent has a purely reactive behaviour and is unaware of other agents. It is only answering to the stimuli it perceives in its own local environment; therefore its behaviour is in a stimulus-response form.

- The environment plays the role of a guide for agent's actions and a medium to store and propagate information about ongoing actions.

\section{Affordance}

This concept was initially introduced by the psychologist of perception James Gibson (1979). An affordance is a possibility of action offered to an actor (human or animal) by an object (or artefact) in its local environment. For instance, in farming systems, actions that are quasi automatically triggered by some perceptions, even not fully instantaneous, may be treated alike: watering thirsty animals, spreading slurry in emergency when its tank is about to overflow, spreading nitrogen fertilizers in case of yellow leaves, treating against pests as soon as they are noticed... The idea is that artefacts located in the actuators' physical context are possibly endowed with the necessary information to trigger actions to perform. 
An affordance can be viewed as triggering perceptionaction-based behaviours. Hence, it can be combined with stigmergy to strengthen the embodiment of information necessary for acting in the environment of reactive agents endowed with simple behaviours. It is so possible to set implicit coordination among agents and, consequently, obtain a coherent global system's behaviour.

\section{MULTIAGENTS REPRESENTATION}

A multiagents system (MAS) is characterized by a set of agents, an environment in which these agents evolve and the interactions between the agents and their environment

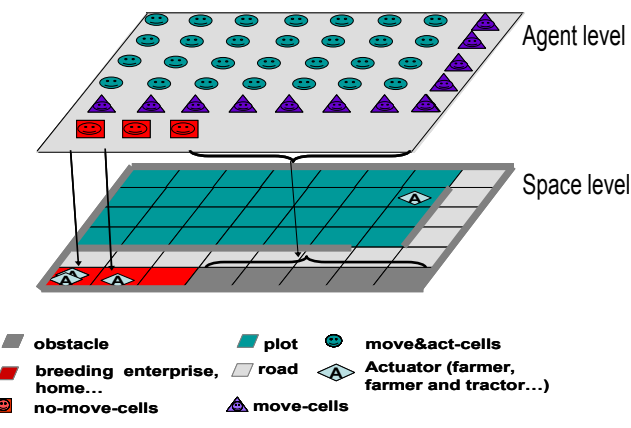

Figure 1. Representation of space as a MAS. and among the agents themselves. An agent is an abstract or real autonomous entity, capable to act by itself and on its environment. Generally in multiagents models such as Brahms (Sierhuis et al., 2006), agents represent humans, animals, robots, machines, etc., that are entities which really act. The environment is considered as an object collection. In keeping with this view that became classical in MAS, the environment in our model is constituted by the union of a physical space and a set of situated objects, namely the objects denoting the resources necessary to agents ('space level' in Fig. 1). However, less classically, we assume that the agents are, in fact, abstract autonomous entities embodied within the environment where they exert locally a control on the situated objects it includes. Therefore, all real-world actors (humans, machines, etc.) are represented by situated objects used as resources by those situated virtual agents to perform actions. This conception seemed to us the most coherent with the theories of situated action, stigmergy, affordances presented above, where the environment and artefacts play the main role and, even it may seem a bit caricatural, 'actors' are actually guided, afforded, conducted... (caricaturizing: manipulated) by the environmental settings they belong to.

The physical space in farming systems holds crop plots, roads, breeding enterprises, buildings (warehouses, machinery depots, etc.), material stocks, obstacles (hedges, ditches), etc. Among the resources we distinguished between three categories: "inputs" (animal feeds, fertilizers...), "equipment" (tractors, ploughs...) and "operators" (farmer, worker). These resources are also represented by objects. So, we defined two object classes, passive objects (e.g. inputs) and actionable objects (e.g. equipment, operator). We denote all the actionable objects by the term "actuator".

\subsection{Actuators}

We introduce the term actuator to make clearly the difference with the concept of agent. Actuators are the entities (actors, machines, etc.) which carry out the actions (tillage, harvest, etc.). They are not autonomous in the sense they cannot take an initiative, neither to act nor to perceive their environment, and they are not endowed with reasoning or decision capacities. An actuator is thus viewed as a specific resource used to achieve an action. Within the framework of farming systems an actuator maybe the conjunct of a tractor with a driver and equipped with a tool determining the type of action it may be ordered to perform (a plough for tillage, a trailer to carry goods, a tanker to transport liquids...). Actuators are considered, at the same time, as artefacts that may afford a cell-agent (cf. $\S 3.2$.) to act and resources to perform an action. An actuator is represented by an object, i.e. a computational entity, encapsulating attributes (namely defining its state), capable to execute methods (of which actions) and communicating by message passing to other objects. For instance, a tractor (actuator) can have the following attributes: identifier, maximum and current speeds, accumulated covered distance, moving direction, etc.

\subsection{Multiagents system architecture}

Space is represented by a discrete regular grid of square cells (Fig. 1). Each cell is controlled by an abstract agent called "cell-agent". The actions performed by a cell-agent differ according to the type of abilities it is endowed. We distinguished three types (Fig. 1):

- Cells where, at the granularity of representation of the system, the activities performed by actuators are considered as immobile (e.g. within a livestock house, a greenhouse, ...); we call them "no-move-cells".

- Cells where the only activity performed is moving (e.g. transport on a road); we call them "move-cells".

- Cells where the activities are necessarily performed while moving (e.g. spreading manure on land or harvesting a plot with a tractor); we call them "move\&act-cells". 


\subsection{Move-cell agent}

The role of this agent is to trigger the action "move" of an actuator. If an actuator is on its cell and if it perceives a request of a nearby cell for using the same actuator, then he may decide (e.g. when the ongoing work is completed) to order the actuator to activate the action "move" indicating it the destination cell.

The request of any cell-agent is materialized by a track made with marks (e.g. flags) the agent deposits on its cell when it is afforded by an actuator. So, both the affordances and the marks (mimicking pheromones of ant colonies) have a semantic. The semantic of affordances is the actions they afford, i.e. they offer the agents to do. For example, the action of ploughing requires a plough hitched to a tractor together with a driver. If one of these elements lacks there will have no affordance for ploughing. The semantic of marks is to propagate some request from one agent to other agents, for example the need of an actuator.

\subsection{No-move-cell and move\&act-cell agents}

These agents' function is to perform agricultural activities such as ploughing, feeding animals, spreading fertilizer, etc. They are endowed with two rule bases necessary for executing any action: starting/ending rules and continuation rules.

\section{Starting/ending rules}

These are rules necessary to define the temporal bounds of actions: i.e. their start and end dates (Guerrin, 2009). The conjunction of conditions required for triggering or stopping an action are denoted $P_{A}(t)$ (see Eq. 2 ). One can find for example cultural calendars, specifying the earliest and latest dates of the tasks to be performed on crops, along with the resources that must be available. For example, spreading slurry requires, in addition to be within a specific period of the year, dry weather, a tractor with a driver and a tanker equipped with spreader, enough manure in stock, working time,... The stopping conditions may be when the required dose of slurry has been spread and/or all the field surface is covered.

\section{Continuation rules}

This base contains the conditions that must hold for continuing an ongoing action. In this base are stored the conditions $C_{A}(t)$ (see Eq. 1). For example, the occurrence of a rainfall, a shortage of slurry, a tractor breakdown, a more urgent task requiring resources (tractor, driver,...), etc., will falsify the conditions listed above and so the action will be interrupted. In some conditions, it can be resumed, if the triggering conditions are met again later, but not necessarily (e.g. if the interruption led the current time out of the time range specified by the cultural calendar). Note that distinguishing between these two propositions allows the conditions to starting, ending (by Eq. 2) or continuing (by Eq. 1) an action to be different although, evidently, there may be overlapping, as in the example above.

\section{Behaviour of no-move- (or movedact-) cell-agent}

The behaviour of a no-move-cell and move\&act-cell agent is simple. It just reacts to the affordances and tracks it finds by selecting in its starting/ending rules the appropriate action to perform. When a no-move- (or move\&act-) cell-agent perceives an affordance (e.g. from an actuator) in a nearby cell, it checks its action starting/ending rules. If the conditions are met for an action, then the agent shows its interest for this affordance by deposing a track on its cell. We assign each track a priority. If two cells put down tracks requesting the same actuator, the cell with higher priority gets it from the cell with lower priority. Note that priorities are fixed according to the importance of an action compared with concurrent ones. For example, let us suppose that a move\&act-cell-agent deposits a track to inform that it needs the farmer to disinfect the soil and another cell puts down a track to inform that it is time to feed animals. Assuming the farmer is on the move\&act-cell, this cell will send him to the no-move-cell where feeding animals is required.

An action can be interrupted during its execution whenever its continuation conditions are no more verified. In that case, the agent controlling the cell where it stands, sends the actuator towards another cell while depositing a track. When an agent receives an actuator, if it perceives a track on the precedent cell then it deposits a similar track on its own cell. This is to keep track and propagate the information that an action $A_{\mathrm{i}}$ on a cell $C_{\mathrm{i}}$ was not completed. This let establishing a mark trail allowing the actuator to come back to the cell $C_{\mathrm{i}}$ when the conditions will again be satisfied.

When the conditions $C_{\mathrm{A}}$ necessary for executing an action are again verified, the agent strengthens the tracks on its cell. When a nearby cell perceives this marking intensification, it strengthens in its turn the track being on its cell if it exists. This way, the information propagates up to the cell which possesses the actuator. Hav- 
ing finished its current action, the actuator will be sent by the cell-agent where it is located back to the former cell to complete the interrupted action. After following the mark trail, when the actuator arrives on the cell where lies the interrupted action, the cell-agent evaporates the track, which incites on its turn each agent marked by the same track to evaporate it.

\section{APPLICATION EXAMPLE}

In this section we describe how it is dealt with some specific situations in our multiagents system. We consider a farming system composed of a crop plot, roads, breeding enterprises, warehouses, machinery depots in which a farmer works. The situations list is the following:

1. When a working day begins, how does the farmer (actuator) get out of its home?

2. How does an actuator find its way towards the livestock building?

We assume taking care of livestock (e.g. feeding animals) has higher priority over other actions, e.g. harvesting that may be delayed a bit.

\section{Situation 1}

Generally, a famer works according to specific time slots. He begins working early in the morning and takes a lunch break. In our model, the agent that controls the home cell is endowed with a clock that rings at fixed periods: (1) at 6:00 am when the working day begins; (2) at 1:00 pm when it is time for break; (3) at 2:00 pm at the break end (4) at 6:00 pm when the working day finishes. For the first and third cases, the actuator must get out of its home. For the second and fourth cases the actuator must be led to return back home. Due to lack of space we treat here only the first case. We consider 2 space configurations described in Fig. 2, left and right respectively:

(i) The home $\mathrm{H}$ is lined by a road cell $\mathrm{R}$, a machinery depot MD and an obstacle cell, the breeding enterprise is farther (Fig. 2 Left);

(ii) The cells nearby the home $\mathrm{H}$ are the breeding enterprise $\mathrm{BE}$, the machinery depot $\mathrm{MD}$ and a road

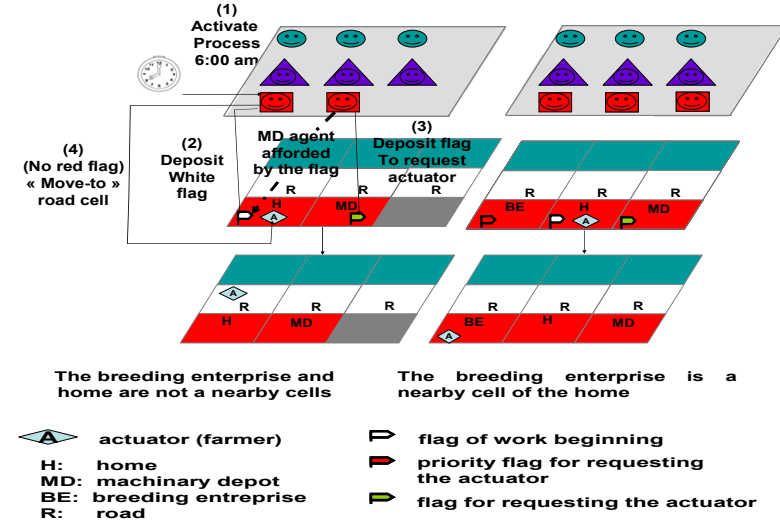

Figure 2. The MAS at the beginning of a working day. cell R (Fig. 2 Right).

For example, when the clock triggers at 6:00 am (Fig. 2 Left (1)), the home agent deposits a mark (white flag) on its cell to inform its nearby cells that the actuator (farmer) is available to work (Fig. 2 Left (2)). This flag may afford the nearby cells to start an action that needs this actuator. For example, the flag may afford the machinery depot to ask the actuator to equip it with a tool (e.g. harvester) that allows it to perform an action on the plot or, alternatively, it may afford the breeding enterprise to request the actuator to feed or to milk the cows. If the situation in each cell allows executing the action which needs this actuator (e.g. if the quantity of hay stored in the breeding enterprise is sufficient to feed animals) then the cell-agents express their interest for the actuator by deposing a colored flags (Fig. 2 Left (3)). The color informs the home agent about the level of priority of the action the actuator is asked for. In this example feeding animals has higher priority over equipping the tractor with a tool. So, a red flag is associated to the action of feeding animals and a green flag for equipping the tractor. Hence, if the home agent perceives a red flag in a nearby cell (Fig. 2 Right) then it activates the action "move-to" the actuator towards this cell, else (Fig. 2 Left) even it perceives a green flag, it sends the actuator towards a road-cell (Fig. 2 Left (4)).

\section{Situation 2}

Among the actions with higher priority there are those which take place in the breeding enterprise. The question is how does the actuator find its way towards the breeding enterprise after having left home at 6:00 am? Initially the actuator takes any direction on the road which is indicated by a move-cell-agent (Fig. 3 Step (1)). As long as the actuator does not meet a red flag or an obstacle, it continues moving on the road by inciting every move-cell-agent to make a yellow weighted mark on their cell or reinforce the mark if it exists. 
If the actuator meets an obstacle, the movecell-agent orders it to turn back and does not deposit a mark (Fig. 3 Step (2)). Should the opposite occur, that is if it meets a red flag (e.g. if it is a breeding enterprise cell), it moves towards this cell and performs the action (Fig. 3 Step (3)). Then, to keep track of its road, the actuator returns home by following the mark, while incrementing their weight and changing their colors to white (Fig. 3 Step (4)). Changing the color prevents the actuator from being attracted by the marks after performing the action in the breeding enterprise. At the beginning of the working day, when a move-agent perceives a white flag (Fig. 2

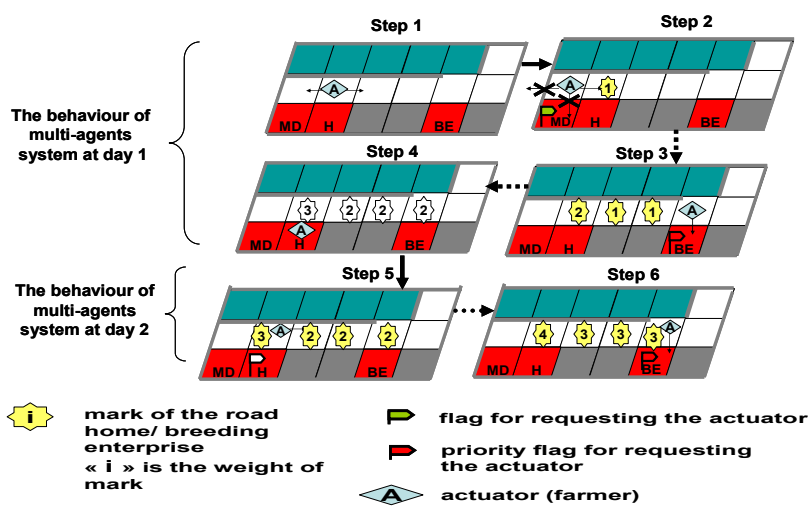

Figure 3. Construction of a mark trail. Left) it changes the mark color to yellow to attract the actuator (Fig. 3 Step (5)). The cells which are marked but are not visited by the actuator every day at the beginning of the work, their agents decrement the weight of marks until they disappear. Note that, in reality, the construction of the road is made after several simulations and incrementing the weight of marks stops after several iterations.

\section{CONCLUSION}

In this paper we have presented a conceptual framework to represent situated action. As this theory argues that action is mainly guided by the agent's environment, we focused on putting the maximum information necessary for the realization of action in the environment. Therefore, we assume agents are embedded within the environment and, in fact, take the role classically devoted to real actors (farmers, workers...) that supposedly rationally decide and act autonomously. Using the stigmergy and affordance concepts, these embedded agents in fact control their environment, dictating the actions to do to passive actuators when the necessary resources are available and propagating information through space.

We tried to apply this conceptual framework to application examples which allowed us to treat some basic elementary situations. Our perspective is to improve this work by dealing with more complex realistic scenarios including several farmers working on various plots, taking care of possibly various livestocks, etc. These farmers may share some equipment or resources and, thus, need to coordinate their activities.

We are aiming now to formalize and implement this conceptual representation to, eventually, build computer models that can simulate real agricultural production systems.

\section{REFERENCES}

Agre, P.E. (1995). Computational Research on Interaction and Agency. Artificial Intelligence, 72(1), 1-52. Bratman, M. E., 1987. Intentions, Plans, and Practical Reason. Harvard University Press: Cambridge, MA. Gibson, J., (1979). The ecological approach to visual perception. Boston, MA, USA: Houghton Mifflin.

Grassé, P.-P., (1959). La reconstruction du nid et les coordinations interindividuelles chez Bellicositermes natalensis et Cubitermes $s p$. La théorie de la stigmergie : essais d'interprétation du comportement des termites constructeurs. Insectes Sociaux., 6: 41-84.

Guerrin, F., 2009. Dynamic simulation of action at operations level. Autonomous agents and multiagents systems, 18(1), 156-185.

Johnston, R.B. (1998). The problem with planning: The significance of theories of activity for operations management, Unpublished PhD Thesis. Monash University, Clayton.

Malone, T.W. and Crowston, K. (1994). The interdisciplinary study of coordination. ACM Computing Surveys, 26 (1), 87-119.

Martin-Clouaire, R. and Rellier, J.P. (2009). Modelling and simulating work practices in agriculture. International Journal on Metadata, Semantics and Ontologies, 4(1-2), 42-53

Sierhuis, M., Clancey, W.J. and Van Hoof, R.J.J. (2006). A multiagents modelling and simulation environment for work processes and practices. International Journal of Simulation and Process Modelling, 3(3), 134-152.

Suchman, L. (1987). Plans and situated actions: The problem of human-machine communication. Cambridge, MA, USA: Cambridge University Press.

Visetti, Y.M. (1989). Compte-rendu: Lucy A. Suchman, Plans and Situated Actions-The Problem of Human/ machine Communication. Cambridge University Press, 1987. Intellectica, 1, 67-96. 